\title{
AN EFFICIENT SYNTHESIS OF NOVEL BIOACTIVE NEW COUMARIN
}

SCHIFF'S BASE.

\author{
Pravin S. Jogi \\ Janata Mahavidyalaya, Chandrapur \\ Corresponding author Email : jogipravin@yahoo.in
}

\begin{abstract}
:
Due to the potential antibacterial activity of Schiff base and coumarin moieties, hybrid compounds containing both structures have been synthesized in good yield. A new efficient method was developed to synthesize Schiff base incorporating coumarin nuclei in excellent yields starting from 7-hydroxy-4-methyl coumarin, Cloroethyl acetate. The method was also successfully applied to the synthesis of coumarin derivatives containing Schiff base. The five novel coumarin bearing different functionalities have been synthesized and confirmed on the basis of their spectral data (1H-, 13C-NMR, UV, IR and mass). They were examined for the first time for antimicrobial activity.
\end{abstract}

\section{Keywords:}

Coumarin, Schiff base, antifungal, Efficient

\section{Introduction:}

Coumarins and their derivatives have been found to exhibit different biological and pharmacological activities [1]. The 4-methylcoumarins have been found to possess anti-inflammatory [2], cholesretic [3], analgesic [4], antispermatogenic [5] and diuretic [6] properties. Apart from the medicinal applications coumarins are also used as sweetener, fixative of perfumes, enhancer of natural oils such as lavender, a food additive in combination with vanillin, a flavour/odour stabilizer in tobacco, an odour masker in paints and rubber. Owning to the widespread applications, synthetic and biological activity evaluation of coumarins and their derivatives has been a subject of intense investigations. The emergence of multidrug-resistant gram-positive bacteria, suchas methicillin- resistant Staphylococcus aureus (MRSA) havemade treatment of infectious diseases difficult and over the last decades, became a serious medical problem. As pathogenic bacteria continuously evolve resistance to 
currently used antibacterial agents, so the discovery of novel and potent antibacterial agents is the best way to overcome bacterial resistance and develop effective therapies [7]. The molecular manipulation of promising lead compounds is still a major line of approach to develop new drugs. It involves an effort to combine the separate pharmacophoric groups of similar activity into one compound, thus making structural changes in the biological activity. Here, we reported the synthesis of new coumarin derivatives containing Schiff base moieties. It is achieved when 7-hydroxy-4-methylcoumarin condensed with Chloroethylacetate to yield 2, which on refluxed with hydrazine hydrate gave 3. The intermediate moiety 3 formed Sciff base 4 on condensation with aromatic aldehydes. Because of the enormous biological importance of coumarin derivatives, the target was to synthesize some coumarin based Sciff's base in a goal to increase their biological relevance so that these compounds could be used for potential antifungal/antibacterial/antiviral activity.

\section{Material and Method:}

1. GENERAL: - All the chemicals and solvents were obtained from E Merck, India (AR grade) and were used without further purification. Melting points were taken in an open capillary tube. IR spectra were recorded on a Shimadzu Dr- 8031 instrument.1H NMR spectra of the synthesized compounds were recorded on a Bruker- Avance (300 MHz), Varian-Gemini (200 MHz) spectrophotometer using $\mathrm{CDCl3}$ solvent and TMS as the internal standard. EIMS spectra were determined on a LCQ ion trap mass spectrometer (Thermo Fisher, San Jose, CA, USA), equipped with an EI source.

2. GENERAL SYNTHESIS 2.1. Synthesis of 7-OH-4-Methylcoumarin: - 7Hydroxy-4- methylcoumarin (1) was prepared in good yield (Scheme.1.) using a Pechmann procedure by condensation of ethylacetoacetate with an equimolar amount of resorcinol in presence of conc [8]. H2SO4. The product was isolated with $95 \%$ yield. The uncorrected $\mathrm{mp}$ was $179-181{ }^{\circ} \mathrm{C}$ and this closely matched the literature value of $180-182{ }^{\circ} \mathrm{C}$. 2.2. Synthesis of 2-(4-methyl-2-oxo-2H- 
chromen-7-yloxy) acetic acid: - A mixture of 7-HO-4- methylcoumarin (1) (0.01 mole), ethyl chloroacetate (0.01 mole) and anhydrous $\mathrm{K} 2 \mathrm{CO} 3$ (0.01 mole) in dry acetone was refluxed on a water bath for 8-9 h. The mixture was then filtered and solvent was removed under reduced pressure. The resulting solid was crystallized from ethanol to afford product (2). 2.3. Synthesis of 2-(4-methyl-2oxo-2H-chromen-7-yloxy) acetohydrazide:-A mixture of compound (2) (0.01 mole) and hydrazine hydrate (0.02 mole) in ethanol was refluxed on a water bath for $9 \mathrm{~h}$. After cooling, the solid that separated was washed with water, dried and recrystallized from ethanol. Needle shaped crystals of (3) were obtained. 2.4. Synthesis of new coumarin derivatives (4a-ae):- A mixture of compound $3(0.01 \mathrm{~mole})$ in ethanol $(30 \mathrm{~mL})$, benzaldehyde a $(0.01 \mathrm{~mole})$ and 1 ml. of glacial acetic acid was refluxed on water bath for $8 \mathrm{~h}$. After cooling, the solvent was removed under reduced pressure and the separated solid was crystallized from methanol to yield 4a. 2.5. In Vitro Antimicrobial Screening:

For biological screening, the agar cup plate method using Hi-Media agar medium was employed to study the antibacterial activity of $4 \mathrm{a}-4 \mathrm{e}$ against Staphylococcus aureus, Proteus vulgaris, Pseudomonas aeruginosa and Escherichia coli. Preparation of nutrient broth, subculture, base layer medium, agar medium and peptone water was done as per the standard procedure.

Each test compound (50 mg) was dissolved in DMF (50 mL, $1000 \mathrm{mg} / \mathrm{mL}$ ), which was used as sample solution. Sample size for all the compounds was fixed at $0.1 \mathrm{~mL}$. Using a sterilized cork borer, cups were scooped out of Agar. Medium contained in a Petri dish which was previously inoculated with the microorganisms. The test compound solution $(0.1 \mathrm{~mL})$ was added in the cups and the Petri dishes were subsequently incubated at $37 \mathrm{oC}$ for $48 \mathrm{~h}$. Ampicillin and Streptomycin were used as reference drugs and DMF as a negative control. Zones of inhibition produced by each compound were measured in millimeters, and the results are listed in Table 1 Table 1 Antibacterial and antifungal bioactivity of compounds 4a-4e Compd. MW g/mol Gram + Gram - Fungal B. subtitis S. aureus S. typhyl E. coli A. niger 4a $336++++++++++++++4$ b 352 
$+++++++++++++4 \mathrm{c} 370+++++++++++++4 \mathrm{~d} 366+++++++++++4 \mathrm{e} 371$

+++++++++++ AMP -++++++++++++ STREP -+++++++++++++ STREP:

Streptomycin; AMP: Ampicillin; - =. Inactive (inhibition zone $<5 \mathrm{~mm}$ ); + = slightly active (inhibition zone $5-10 \mathrm{~mm}$ ); $++=$ moderately active (inhibition zone 10-15 mm); +++ = highly active (inhibition zone $>15 \mathrm{~mm}$. Scheme. 1. 2.6. Spectroscopic characterization of $4 a-4 e$ 1. (E)-N'-benzylidene-2-(4-methyl-2oxo-2H-chromen-7-yloxy) acetohydrazide (4a) :- M.P.: 139; \% yield: 75; IR (KBr, cm-1): 1090 (C-O str.), 1754 (lactone, C=O str.), 1660 (C=N str.), 2958 (-CH3 str.), 3012 (Ar-H str.), 3421 (-NH str. Sec. amine).1HNMR (400 Mz, DMSOd6, ppm): 2.4 (s, 3H, CH3), 6.95-7.83 (m, 8H, Ar-H), 6.22 (s, 1H, vinylic -CH), 11.20 (s, 1H, N-H, Sec. amine), 8.53 (s, 1H, benzyidenium). 13C NMR (DMSOd6, ppm): 125.0 (C1-coumarin), 155.4 (C2-coumarin), 115.6 (C3-coumarin), 123.4 (C4-coumarin), 115.7 (C5-coumarin), 155.8 (C6- coumarin), 160.0 (C7coumarin), 112.5 (C8-coumarin), 152.6 (C9-coumarin), 20.4 (C10-coumarin), 144.9 (CH=N, imine), 173 (amide) , 133.7-128.8 (Phenyl ring). MS (C19H16N2O4): m/z 336 (Mp, 100\%). Elemental analysis: calcd. (found): C, 67.7867 (67.7521), H, 4.7569 (4.7552), N, 8.3246 (8.3224). 2. (E)-N'-(4hydroxybenzylidene)-2-(4-methyl-2-oxo-2H-chromen-7-

yloxy)acetohydrazide(4b) M.P.: 145; \% yield: 75; IR (KBr, cm-1): 1090 (C-O str.), 1720 (lactone, $\mathrm{C}=\mathrm{O}$ str.), 1650 (C=N str.), 2958 (-CH3 str.), 3120 (Ar-H str.), 3421 (-NH str. Sec. amine) 3415 (-OH str.).1HNMR (400 Mz, DMSOd6, ppm): $2.4(\mathrm{~s}, 3 \mathrm{H}, \mathrm{CH} 3), 10.1(\mathrm{~s}, 1 \mathrm{H},-\mathrm{OH}) 6.95-7.83(\mathrm{~m}, 8 \mathrm{H}, \mathrm{Ar}-\mathrm{H}), 6.12$ (s, 1H, vinylic -CH), 11.36 (s, 1H, N-H, Sec. amine), 8.50 (s, 1H, benzyidenium). 13C NMR (DMSO-d6, ppm): 124 (C1-coumarin), 153.4 (C2-coumarin), 113.6 (C3coumarin), 122 (C4-coumarin), 114 (C5-coumarin), 156 (C6- coumarin), 160 (C7-coumarin), 112.5 (C8-coumarin), 152.6 (C9-coumarin), 20.1 (C10coumarin), $144.9(\mathrm{CH}=\mathrm{N}$, imine), 173 (amide) , 131-127 (Phenyl ring). MS (C19H16N2O5): m/z 352 (Mp, 100\%). Elemental analysis: calcd. (found): C, 64.7727 (64.7600), H, 4.5454 (4.5120), N, 7.9545 (7.9445). 3. (E)-N'-(4chlorobenzylidene)-2-(4-methyl-2-oxo-2H-chromen-7-yloxy)acetohydrazide(4c) 
:- M.P.: 152; \% yield: 75; IR (KBr, cm-1): 1090 (C-O str.), 1715 (lactone, $\mathrm{C}=\mathrm{O}$ str.), 1658 (C=N str.), 2958 (-CH3 str.), 3251 (Ar-H str.), 3421 (-NH str. Sec. amine).1HNMR (400 Mz, DMSOd6, ppm): 2.4 (s, 3H, CH3), 6.95-7.83 (m, 7H, Ar-H), 6.31 (s, 1H, vinylic $-\mathrm{CH}), 10.69$ (s, 1H, N-H, Sec. amine), 8.34(s, 1H, benzyidenium). 13C NMR (DMSO-d6, ppm): 125.3 (C1-coumarin), 155.5 (C2coumarin), 116.2 (C3-coumarin), 123.4 (C4-coumarin), 115.7 (C5-coumarin), 155 (C6- coumarin), 161.0 (C7-coumarin), 112.5 (C8-coumarin), 152.6 (C9coumarin), 21(C10-coumarin), $144.9(\mathrm{CH}=\mathrm{N}$, imine), 171 (amide) , 133.7-128.8 (Phenyl ring). MS (C19H15ClN2O4): m/z 370 (Mp, 100\%). Elemental analysis: calcd. (found): C, 61.6216 (61.6120), H, 4.0540 (4.0041), N, 7.5675 (7.5420). 4. (E)-N'-(4-methoxybenzylidene)-2-(4-methyl-2-oxo-2H-chromen-7yloxy)acetohydrazide(4d) :- M.P.: 134; \% yield: 75; IR (KBr, cm-1): 1090 (C-O str.), 1725 (lactone, C=O str.), 1696 (C=N str.), 2958 (-CH3 str.), 3099 (Ar-H str.), 3421 (-NH str. Sec. amine).1HNMR (400 Mz, DMSOd6, ppm): 2.4 (s, 3H, CH3), 3.6 (s, 3H, OCH3), 6.95-7.83 (m, 7H, Ar-H), 6.10 (s, 1H, vinylic - $\mathrm{CH})$, 11.82 (s, 1H, N-H, Sec. amine), 8.4 (s, 1H, benzyidenium). 13C NMR (DMSOd6, ppm): 124.0 (C1-coumarin), 155.9 (C2-coumarin), 115.2 (C3-coumarin), 123 (C4-coumarin), 115.2 (C5-coumarin), 155.7 (C6- coumarin), 161.2 (C7coumarin), 112.1 (C8-coumarin), 152.2 (C9-coumarin), 20.9 (C10-coumarin), $144(\mathrm{CH}=\mathrm{N}$, imine), 174 (amide) , 130-128 (Phenyl ring). MS (C2OH18N2O5): m/z 366 (Mp, 100\%). Elemental analysis: calcd. (found): C, 65.5737 (65.1240), H, 4.9180 (4.8254), N, 7.6502 (7.4510). 5. (E)-N'-(4-

(dimethylamino)benzylidene)-2-(4-methyl-2-oxo-2H-chromen-7-

yloxy)acetohydrazide(4e) :- M.P.: 167; \% yield: 75; IR (KBr, cm-1): 1090 (C-O str.), 1730 (lactone, C=O str.), 1637 (C=N str.), 2958 (-CH3 str.), 3042 (Ar-H str.), 3421 (-NH str. Sec. amine).1HNMR (400 Mz, DMSOd6, ppm): 2.4 (s, 3H, CH3), 2.9(s, 6H, N(CH3)2) 6.95-7.83 (m, 7H, Ar-H), 6.30 (s, 1H, vinylic $-\mathrm{CH})$, 11.12 (s, 1H, N-H, Sec. amine), 8.3 (s, 1H, benzyidenium). 13C NMR (DMSOd6, ppm): 125.0 (C1-coumarin), 155.2 (C2-coumarin), 115 (C3-coumarin), 123.6 (C4-coumarin), 115.1 (C5-coumarin), 155.3 (C6- coumarin), 160.0 (C7- 
coumarin), 112.5 (C8-coumarin), 153.6 (C9-coumarin), 22.4 (C10-coumarin), $144(\mathrm{CH}=\mathrm{N}, \quad$ imine), 171 (amide) , 133.7-128.8 (Phenyl ring). MS (C21H21N3O4): m/z 371 (Mp, 100\%). Elemental analysis: calcd. (found): C, 67.9245 (67.8921), H, 5.6603 (5.4769), N, 11.3207 (11.2905).

\section{Result and Discussion:}

1.SYNTHESIS: - The coumarin was synthesized by the well known Pechmann condensation in quantitative yield. The intermediates 2 and 3 were synthesized by usual reaction with some modification in reaction conditions. Series of five new Schiff bases with coumarin structure $4 \mathrm{a}-4 \mathrm{e}$ have been prepared by simple methodoly reported for first time Scheme-1.

2.SPECTRAL STUDY: -Structure of the synthesized compoundswas established on the basis of IR, 1H NMR, 13C NMR and Mass spectral data. The general spectral characters of $4 \mathrm{a}-4 \mathrm{e}$ showed absorption bands ranging from 1696-1637 $\mathrm{cm}-1$ for imine $(-\mathrm{C}=\mathrm{N})$ formation and $1754-1715 \mathrm{~cm}-1$ for $(\mathrm{C}=\mathrm{O})$ of coumarin.

The $1 \mathrm{H}-\mathrm{NMR}$ spectra were recorded in DMSO-d6 at room temperature using TMS as internal standard. In particular, it must be pointed out that $1 \mathrm{H}$ NMR of compounds $4 \mathrm{a}-4 \mathrm{e}$ showed presence of a singlet between $\delta 8.3-8.5 \mathrm{ppm}$ indicated the formation of imine $(-\mathrm{CH}=\mathrm{N}-)$ by simple condensation process.

Further characteristic peaks at $\delta 2.4 \mathrm{ppm}$ indicated the presence of 4-CH3 group of coumarin ring in their structure. All synthesized compounds have showed prominent peak around $\delta 11 \mathrm{ppm}$ confirmed secondary amine structural unit. The compounds $4 \mathrm{a}-4 \mathrm{e}$ showed prominent singlet around $\delta$

$6.22 \mathrm{ppm}$ for 3rd proton of coumarin and multiplet at $\delta 6.95-7.83 \mathrm{ppm}$ for aromatic protons. The presence of lactone carbonyl group is in agreement with their IR value. The IR peak in between 3300- 3100 corresponds to aromatic hydrogen stretching. All other peaks in the spectra are in well agreement with the contents of functionalities in the synthesized coumarin Schiff base derivatives. The other signals and peaks of $1 \mathrm{H}-\mathrm{NMR}$ and IR are in complete agreement with the assigned structures. 13C NMR spectral data for the title 
compounds most characteristic peak around $\delta 144.9$ and $173 \mathrm{ppm}$ indicated the formation Schiff base linkage and amide moiety in molecule. Electron impact mass spectra showed an accurate molecular ion peaks which are in agreement with molecular mass of synthesized compounds. All the compounds have given the satisfactory elemental analysis. The synthesized compounds were evaluated for in vitro antibacterial and antifungal activity against various gram-positive, gram-negative bacteria and fungal strains using agar cup plate method. The results are shown in table- 1 suggest that compounds $4 \mathrm{a}, 4 \mathrm{~b}$ and $4 \mathrm{c}$ are highly active against bacterial strain as well as fungi. Compounds $4 \mathrm{~d}$ and $4 \mathrm{e}$ are moderately active against bacterial strain but highly active against fungi

\section{Conclusion:}

In this paper we report the synthesis and antimicrobial activity of novel series of Schiff base. The derivatives were prepared by condensation between 7 hydroxy-4-methylcoumarin derivatives with various substituted aromatic aldehydes to obtain schiff bases compounds $(4 a-4 e)$. The preliminary in vitro antimicrobial activity of these novel series of derivative has evidenced that derivatives are more active than that of schiff bases. Further studies are required for the detailed mechanism of action of these derivatives. Some of Compounds were found to be equally potent as compared to Ampicillin and Streptomycin.

\section{Acknowledgement:}

Corresponding author is thankful to the Secretary, CSPM, Chandrapur \& Principal, J.M.V. Chandrapur. 


\section{Reference:}

Egan D., Kennedy R.O., Moran E., Cox D. and Prosser E. (1990): Drug Metab, Rev., 22, Pp 503-529.

Bhalla M., Nathani P.K., Kumar A., Bhalla T.N. and Shanker K. (1992): J. Indian Chem., 31, (B), Pp 183-186.

Takeda S. and Aburada M. (1981): J. Pharmacobiodyn., 4, Pp 724-729.

Yang C.H., Chiang C., Liu K., Perg S., Wang R. and Yao Huseh Tung Pao., (1980): 15 Pp48-54.

Tyagi A., Dixit V.P. and Joshi B.C., Naturwissenschaften., (1980): 67, Pp 104109.

Deana A.J., Med. Chem. (1983): 26, Pp 580-585.

Coleman K., (2004): Recent advances in the treatment of Gram-positive infections., Drug Discov. Today Ther. Strateg., 1, Pp 455-460.

Jogi, P.S., Meshram J.S. and Shaikh J. (2013): Synthesis and Evaluation of Antimicrobial Activity of NovelOxazine Derivatives using Betti"s Protocol., Letters in Drug Design \& Discovery, Benthum Science, 10, Pp255-264. 